\title{
INFINITE MATRICES, WAVELET COEFFICIENTS AND FRAMES
}

\section{N. A. SHEIKH and M. MURSALEEN}

Received 2 December 2003

We study the action of $A$ on $f \in L^{2}(\mathbb{R})$ and on its wavelet coefficients, where $A=\left(a_{l m j k}\right)_{l m j k}$ is a double infinite matrix. We find the frame condition for $A$-transform of $f \in L^{2}(\mathbb{R})$ whose wavelet series expansion is known.

2000 Mathematics Subject Classification: 42C15, 41A17, 42C40.

1. Introduction. The notation of frame goes back to Duffin and Schaeffer [7] in the early 1950s to deal with the problems in nonharmonic Fourier series. There has been renewed interest in the subject related to its role in wavelet theory. For a glance of the recent development and work on frames and related topics, see [3, 4, 5, 6, 9]. In this note, we will use the regular double infinite matrices (see $[9,10])$ to obtain the frame conditions and wavelet coefficients.

2. Notations and known results. $\mathbb{N}$ is the set of positive integers, $\mathbb{Z}$ is the set of integers, $\mathbb{R}$ is the set of real numbers. The space $L^{2}(\mathbb{R})$ of measurable function $f$ is defined on the real line $\mathbb{R}$, that satisfies

$$
\int_{-\infty}^{\infty}|f(x)|^{2} d x<\infty
$$

The inner product of two square integrable functions $f, g \in L^{2}(\mathbb{R})$ is defined as

$$
\begin{gathered}
\langle f, g\rangle=\int_{-\infty}^{\infty} f(x) \overline{g(x)} d x, \\
\|f\|^{2}=\langle f, f\rangle^{1 / 2} .
\end{gathered}
$$

Every function $f \in L^{2}(\mathbb{R})$ can be written as

$$
f(x)=\sum_{j, k \in Z} C_{j, k} \psi_{j, k}(x)
$$

This series representation of $f$ is called wavelet series. Analogous to the notation of Fourier coefficients, the wavelet coefficients $C_{j, k}$ are given by

$$
\begin{gathered}
C_{j, k}=\int_{-\infty}^{\infty} f(x) \overline{\psi_{j, k}(x)} d x=\left\langle f, \psi_{j, k}\right\rangle, \\
\psi_{j, k}=2^{j / 2} \psi\left(2^{j} x-k\right) .
\end{gathered}
$$


Now, if we define an integral transform

$$
\left(W_{\psi} f\right)(b, a)=|a|^{-1 / 2} \int_{-\infty}^{\infty} f(x) \overline{\psi\left(\frac{x-b}{a}\right)} d x, \quad f \in L^{2}(\mathbb{R}),
$$

then the wavelet coefficients become

$$
C_{j, k}=\left(W_{\psi} f\right)\left(\frac{k}{2^{j}}, \frac{1}{2^{j}}\right) .
$$

A sequence $\left\{x_{n}\right\}$ in a Hilbert space $H$ is a frame if there exist constants $c_{1}$ and $c_{2}$, $0<c_{1} \leq c_{2}<\infty$, such that

$$
c_{1}\|f\|^{2} \leq \sum_{n \in Z}\left|\left\langle f, x_{n}\right\rangle\right|^{2} \leq c_{2}\|f\|^{2},
$$

for all $f \in H$. The supremum of all such numbers $c_{1}$ and infimum of all such numbers $c_{2}$ are called the frame bounds of the frame. The frame is called tight frame when $c_{1}=c_{2}$ and is called normalized tight frame when $c_{1}=c_{2}=1$. Any orthonormal basis in a Hilbert space $H$ is a normalized tight frame. The connection between frames and numerically stable reconstruction from discretized wavelet was pointed out by Grossmann et al. [8]. In 1985, they defined that a wavelet function $\psi \in L^{2}(\mathbb{R})$, constitutes a frame with frame bounds $c_{1}$ and $c_{2}$, if any $f \in L^{2}(\mathbb{R})$ such that

$$
c_{1}\|f\|^{2} \leq \sum_{j, k \in z}\left|\left\langle f, \psi_{j, k}\right\rangle\right|^{2} \leq c_{2}\|f\|^{2} .
$$

Again, it is said to be tight if $c_{1}=c_{2}$ and is said to be exact if it ceases to be frame by removing any of its elements. There are many examples proposed by Daubechies et al. [6]. For further details, one can refer to [1, 5,6]. Chui and Shi [2] proved that $\left\{\psi_{j, k}\right\}$ is a frame for $L^{2}(\mathbb{R})$ with bounds $c_{1}$ and $c_{2}$, if for some $a>1$ and $b>0$, the Fourier transform $\hat{\psi}$ satisfies

$$
c_{1} \leq \frac{1}{b} \sum_{j \in Z}\left|\hat{\psi}\left(a^{j} w\right)\right|^{2} \leq c_{2} \text { a.e., }
$$

for some constants $c_{1}$ and $c_{2}$. By integrating each term in

$$
\frac{c_{1}}{|w|} \leq \frac{1}{b} \sum_{j \in Z} \frac{\left|\hat{\psi}\left(a^{j} w\right)\right|^{2}}{|w|} \leq \frac{c_{2}}{|w|}
$$

over $1 \leq|w| \leq a$, we have

$$
2 c_{1} \log a \leq \frac{1}{b} \sum_{j \in Z} \int_{1 \leq|w| \leq a} \frac{\left|\hat{\psi}\left(a^{j} w\right)\right|^{2}}{|w|} d w \leq 2 c_{2} \log a,
$$

which immediately yields

$$
c_{1} \leq \frac{1}{2 b \log a} \int_{-\infty}^{\infty} \frac{\left|\hat{\psi}\left(a^{j} w\right)\right|^{2}}{|w|} d w \leq c_{2} .
$$


The above condition known as compactibility condition was also observed by Daubechies [4] by using techniques from trace class operators. The above constants were given by frame bounds, see [2].

Let $A=\left(a_{m n j k}\right)$ be a double infinite matrix of real numbers. Then, $A$-transform of a double sequence $x=\left(x_{j k}\right)$ is

$$
\sum_{j=0}^{\infty} \sum_{k=0}^{\infty} a_{m n j k} x_{j k},
$$

which is called $A$-means or $A$-transform of the sequence $x=\left(x_{i j}\right)$. This definition is due to Móricz and Rhoades [9].

A double matrix $A=\left(a_{m n j k}\right)$ is said to be regular (see [10]) if the following conditions hold:

(i) $\lim _{m, n \rightarrow \infty} \sum_{j, k=0}^{\infty} a_{m n j k}=1$,

(ii) $\lim _{m, n \rightarrow \infty} \sum_{j=0}^{\infty}\left|a_{m n j k}\right|=0,(k=0,1,2, \ldots)$,

(iii) $\lim _{m, n \rightarrow \infty} \sum_{k=0}^{\infty}\left|a_{m n j k}\right|=0,(j=0,1,2, \ldots)$,

(iv) $\|A\|=\sup _{m, n>0} \sum_{j, k=0}^{\infty}\left|a_{m, n}\right|<\infty$.

Either of conditions (ii) and (iii) implies that

$$
\lim _{m, n \rightarrow \infty} a_{m n j k}=0 .
$$

In this note, we establish the frame condition by using $A$-transform of nonnegative regular matrix, also we find action of the matrix $A$ on wavelet coefficients.

3. Main results. In this section, we prove the following theorems.

THEOREM 3.1. Let $A=\left(a_{i l j k}\right)$ be a double nonnegative regular matrix. If

$$
f(x)=\sum_{j, k \in Z} C_{j, k} \psi_{j, k}(x)
$$

is a wavelet expansion of $f \in L^{2}(\mathbb{R})$ with wavelet coefficients

$$
C_{j, k}=\int_{-\infty}^{\infty} f(x) \overline{\psi_{j, k}(x)} d x=\left\langle f, \psi_{j, k}\right\rangle,
$$

then the frame condition for A-transform of $f \in L^{2}(\mathbb{R})$ is

$$
c_{1}\|f\|^{2} \leq \sum_{i, l \in Z}\left|\left\langle A f, \psi_{i, l}\right\rangle\right|^{2} \leq c_{2}\|f\|^{2},
$$

where $A f$ is the A-transform of $f$ and $0<c_{1} \leq c_{2}<\infty$.

THEOREM 3.2. If $C_{j, k}$ are the wavelet coefficients of $f \in L^{2}(\mathbb{R})$, that is, $C_{j, k}=\left\langle f, \psi_{j, k}\right\rangle$, then the $d_{l, m}$ are the wavelet coefficients of $A f$, where $\left\{d_{l, m}\right\}$ is defined as the $A$ transform of $\left\{C_{j, k}\right\}$ by

$$
d_{l, m}=\sum_{j, k=-\infty}^{\infty} a_{l m j k} C_{j k} .
$$


THEOREM 3.3. Let $A=\left(a_{l m j k}\right)$ be a double nonnegative matrix whose elements are $\left\langle\psi_{j, k}, \psi_{l, m}\right\rangle$. Then, $\left\{\psi_{j, k}\right\}$ constitutes a frame of $L^{2}(\mathbb{R})$ if and only if $\left\{\psi_{l, m}\right\}$ constitutes a frame of $L^{2}(\mathbb{R})$, where $C_{j, k}=\left\langle f, \psi_{j, k}\right\rangle$ and $d_{l, m}=\left\langle f, \psi_{l, m}\right\rangle$.

Proof of Theorem 3.1. We can write

$$
f(x)=\sum_{j, k \in Z}\left\langle f, \psi_{j, k}\right\rangle \psi_{j, k}
$$

If we take $A$-transform of $f$, we get

$$
A f(x)=\sum_{i, l \in Z}\left\langle A f, \psi_{i, l}\right\rangle \psi_{i, l}
$$

and therefore

$$
\begin{aligned}
\sum_{i, l \in Z}\left|\left\langle A f, \psi_{i, l}\right\rangle\right|^{2} & \leq \sum_{i, l \in Z} \int_{-\infty}^{\infty}|A f(x)|^{2}\left|\overline{\psi_{i, l}(x)}\right|^{2} d x \\
& \leq\|A\|^{2}\|f\|_{2}^{2} \sum_{i, l \in Z}\left\|\psi_{i, l}\right\|_{2}^{2} .
\end{aligned}
$$

Since $A$ is regular matrix and $\left\|\psi_{i, l}\right\|_{2}=1$, therefore

$$
\sum_{i, l \in Z}\left|\left\langle A f, \psi_{i, l}\right\rangle\right|^{2} \leq c_{2}\|f\|_{2}^{2}
$$

where $c_{2}$ is positive constant.

Now, for any arbitrarily $f \in L^{2}(\mathbb{R})$, define

$$
\tilde{f}=\left[\sum_{i, l \in Z}\left|\left\langle A f, \psi_{i, l}\right\rangle\right|^{2}\right]^{-1 / 2} f .
$$

Clearly,

$$
\left\langle A \tilde{f}, \psi_{i, l}\right\rangle=\left[\sum_{i, l \in Z}\left|\left\langle A f, \psi_{i, l}\right\rangle\right|^{2}\right]^{-1 / 2}\left\langle A f, \psi_{i, l}\right\rangle,
$$

then

$$
\sum_{i, l \in Z}\left|\left\langle A f, \psi_{i, l}\right\rangle\right|^{2} \leq 1
$$


Hence, if there exists $\alpha$ a positive constant, then

$$
\begin{gathered}
\|A \tilde{f}\|_{2}^{2} \leq \alpha \\
{\left[\sum_{i, l \in z}\left|\left\langle A f, \psi_{i, l}\right\rangle\right|^{2}\right]^{-1}\|A f\|_{2}^{2} \leq \alpha .}
\end{gathered}
$$

Since $A$ is regular, we have

$$
\left[\sum_{i, l \in z}\left|\left\langle A f, \psi_{i, l}\right\rangle\right|^{2}\right]^{-1}\|f\|_{2}^{2} \leq \alpha_{1}\left(=\frac{\alpha}{\|A\|^{2}}\right),
$$

where $\alpha_{1}$ is another positive constant. Therefore,

$$
c_{1}\|f\|_{2}^{2} \leq \sum_{i, l \in z}\left|\left\langle A f, \psi_{i, l}\right\rangle\right|^{2},
$$

where $c_{1}=\alpha>0$.

Combining (3.8) and (3.14), we have

$$
c_{1}\|f\|_{2}^{2} \leq \sum_{i, l \in z}\left|\left\langle A f, \psi_{i, l}\right\rangle\right|^{2} \leq c_{2}\|f\|_{2}^{2} .
$$

This completes the proof.

Proof OF TheOrem 3.2. We can write

$$
\begin{aligned}
\left\langle A f, \psi_{j, k}\right\rangle & =\int_{-\infty}^{\infty} A f(x) \overline{\psi_{l, m}(x)} d x \\
& =\int_{-\infty}^{\infty} \sum_{j, k=-\infty}^{\infty} a_{l m j k} c_{j, k} \psi_{j, k}(x) \overline{\psi_{l, m}(x)} d x .
\end{aligned}
$$

Now,

$$
\begin{aligned}
\sum_{l, m=-\infty}^{\infty}\left\langle A f, \psi_{l, m}\right\rangle \psi_{l, m} & =\sum_{l, m=-\infty}^{\infty} \int_{-\infty}^{\infty} \sum_{j, k-\infty}^{\infty} a_{l m j k} c_{j, k} \psi_{j, k}(x) \psi_{l, m}(x) \overline{\psi_{l, m}(x)} d x \\
& =\sum_{l, m=-\infty}^{\infty} d_{l, m} \psi_{l, m} \int_{-\infty}^{\infty}\left\|\psi_{l, m}(x)\right\|_{2}^{2} \\
& =\sum_{l, m=-\infty}^{\infty} d_{l, m} \psi_{l, m} .
\end{aligned}
$$

Therefore,

$$
\sum_{l, m=-\infty}^{\infty} d_{l, m} \psi_{l, m}=\sum_{l, m=-\infty}^{\infty}\left\langle A f, \psi_{l, m}\right\rangle \psi_{l, m} .
$$

This implies that $d_{l, m}$ are wavelet coefficients of $A f$. 
Thus,

$$
d_{l, m}=\left\langle f, \psi_{l, m}\right\rangle
$$

This completes the proof.

Proof of TheOrem 3.3. We observe that

$$
\begin{aligned}
a_{l m j k} C_{j, k} & =\left\langle\psi_{j, k}, \psi_{l, m}\right\rangle\left\langle f, \psi_{j, k}\right\rangle \\
& =\int_{-\infty}^{\infty} \psi_{j, k}(x) \overline{\psi_{l, m}(x)} d x \int_{-\infty}^{\infty} f(x) \overline{\psi_{j, k}(x)} d x \\
& =\int_{-\infty}^{\infty} f(x) \overline{\psi_{l, m}(x)} d x \int_{-\infty}^{\infty} \psi_{j, k}(x) \overline{\psi_{j, k}(x)} d x \\
& =\int_{-\infty}^{\infty} f(x) \overline{\psi_{l, m}(x)} d x \\
& =\left\langle f, \psi_{l, m}\right\rangle,
\end{aligned}
$$

that is, $a_{l m j k} C_{j, k}=d_{l, m}$.

Now,

$$
\begin{aligned}
& \sum_{l, m}\left|d_{l, m}\right|^{2}=\sum_{l, m}\left|a_{l m j k} C_{j, k}\right|^{2}=\sum_{l, m}\left|\left\langle f, \psi_{l, m}\right\rangle\right|^{2} \\
&=\frac{1}{(2 \pi)^{2}} \sum_{l, m}\left|\left\langle\hat{f}, \hat{\psi}_{l, m}\right\rangle\right|^{2}, \\
& \frac{1}{(2 \pi)^{2}} \sum_{l, m}\left|\int_{0}^{2 \pi} \sum_{p=-\infty}^{\infty} \hat{f}(w+2 \pi p) \overline{\hat{\psi}(w+2 \pi p)} e^{i l m w} d w\right|^{2} \\
&=p \\
&=\frac{1}{2 \pi} \int_{0}^{2 \pi}\left|\sum_{p=-\infty}^{\infty} \hat{f}(w+2 \pi p) \overline{\hat{\psi}(w+2 \pi p)} d w\right|^{2},
\end{aligned}
$$

by Parseval's formula for trigonometric Fourier series.

Now

$$
\begin{aligned}
\left|\sum_{p=-\infty}^{\infty} \hat{f}(w+2 \pi p) \hat{\psi}(w+2 \pi p)\right|^{2} & =\left(\sum_{p=-\infty}^{\infty} \hat{f}(w+2 \pi p) \overline{\hat{\psi}(w+2 \Pi p)}\right) \\
& \times\left(\sum_{q=-\infty}^{\infty} \overline{\hat{f}(w+2 \pi q)} \hat{\psi}(w+2 \pi q)\right) .
\end{aligned}
$$

Let $f(w)=\sum_{q=-\infty}^{\infty} \overline{\hat{f}(w+2 \pi q)} \hat{\psi}(w+2 \pi q)$. 
Therefore,

$$
\begin{aligned}
p & =\frac{1}{2 \pi} \int_{0}^{2 \pi}\left|\sum_{p=-\infty}^{\infty} \hat{f}(w+2 \pi p) \overline{\hat{\psi}(w+2 \pi p)} d w\right|^{2} \\
& =\frac{1}{2 \pi}\left(\int_{0}^{2 \pi} \sum_{p=-\infty}^{\infty} \hat{f}(w+2 \pi p) \overline{\hat{\psi}(w+2 \pi p)} d w F(w) d w\right) \\
& =\frac{1}{2 \pi}\left(\int_{-\infty}^{\infty} \hat{f}(w) \overline{\hat{\psi}(w)} F(w) d w\right) \\
& =\frac{1}{2 \pi}\left\{\sum_{q=-\infty}^{\infty} \int_{-\infty}^{\infty} \hat{f}(w) \overline{\hat{\psi}(w)} \hat{\hat{f}(w+2 \pi q)} \hat{\psi}(w+2 \pi q) d w\right\} \\
& =\frac{1}{2 \pi} \int_{-\infty}^{\infty} \hat{f}(w) \overline{\hat{\psi}(w)} \hat{\hat{f}(w)} \hat{\psi}(w) d w \\
& =\frac{1}{2 \pi} \int_{-\infty}^{\infty}|\hat{f}(w)|^{2}|\hat{\psi}(w)|^{2} d w \\
& =\frac{1}{2 \pi} \int_{-\infty}^{\infty}|\hat{f}(w)|^{2} d w \\
& =\|f\|_{2}^{2},
\end{aligned}
$$

that is,

$$
\sum_{l, m}\left|d_{l m}\right|^{2}=\|f\|_{2}^{2}, \quad f \in L^{2}(\mathbb{R}) .
$$

Therefore, for a regular matrix $A=\left(a_{l m j k}\right)$, we have

$$
c_{1}\|f\|_{2}^{2} \leq \sum_{l, m}\left|d_{l m}\right|^{2} \leq c_{2}\|f\|_{2}^{2}
$$

if and only if

$$
c_{1}^{\prime}\|f\|_{2}^{2} \leq \sum_{j, k}\left|c_{j k}\right|^{2} \leq c_{2}^{\prime}\|f\|_{2}^{2},
$$

where, $0 \leq c_{1}^{\prime}, c_{2}^{\prime}<\infty$. This completes the proof.

\section{REFERENCES}

[1] C. K. Chui, An Introduction to Wavelets, Wavelet Analysis and Its Applications, vol. 1, Academic Press, Massachusetts, 1992.

[2] C. K. Chui and X. L. Shi, Inequalities of Littlewood-Paley type for frames and wavelets, SIAM J. Math. Anal. 24 (1993), no. 1, 263-277.

[3] I. Daubechies, Orthonormal bases of compactly supported wavelets, Comm. Pure Appl. Math. 41 (1988), no. 7, 909-996.

[4] - The wavelet transform, time-frequency localization and signal analysis, IEEE Trans. Inform. Theory 36 (1990), no. 5, 961-1005.

[5] _ Ten Lectures on Wavelets, CBMS-NSF Regional Conference Series in Applied Mathematics, vol. 61, Society for Industrial and Applied Mathematics (SIAM), Pennsylvania, 1992. 
[6] I. Daubechies, A. Grossmann, and Y. Meyer, Painless nonorthogonal expansions, J. Math. Phys. 27 (1986), no. 5, 1271-1283.

[7] R. J. Duffin and A. C. Schaeffer, A class of nonharmonic Fourier series, Trans. Amer. Math. Soc. 72 (1952), 341-366.

[8] A. Grossmann, J. Morlet, and T. Paul, Transforms associated to square integrable group representations. I. General results, J. Math. Phys. 26 (1985), no. 10, 2473-2479.

[9] F. Móricz and B. E. Rhoades, Comparison theorems for double summability methods, Publ. Math. Debrecen 36 (1989), no. 1-4, 207-220.

[10] G. M. Robinson, Divergent double sequences and series, Trans. Amer. Math. Soc. 28 (1926), 50-73.

N. A. Sheikh: Department of Mathematics, National Institute of Technology, Srinagar, Kashmir 190006, Jammu and Kashmir, India

E-mail address: neyaznit@yahoo.co.in

M. Mursaleen: Department of Mathematics, Aligarh Muslim University, Aligarh 202002, Uttar Pradesh, India

Current address: Department of Mathematics, Faculty of Science, P.O. Box 80203, King Abdul Aziz University, Jeddah, Kingdom of Saudi Arabia

E-mail address: mursa7een@postmark. net 


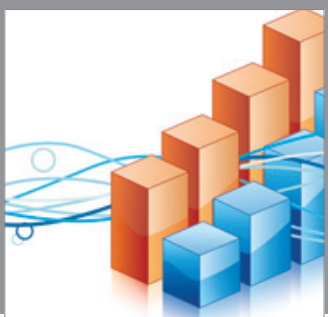

Advances in

Operations Research

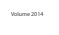

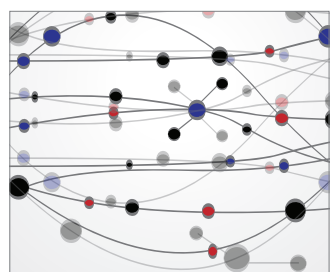

\section{The Scientific} World Journal
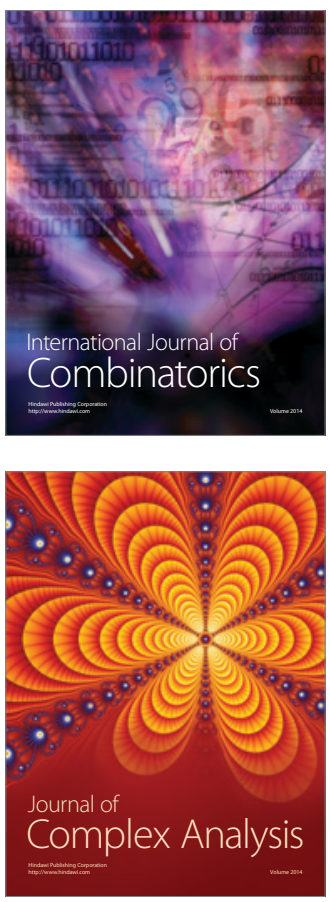

International Journal of

Mathematics and

Mathematical

Sciences
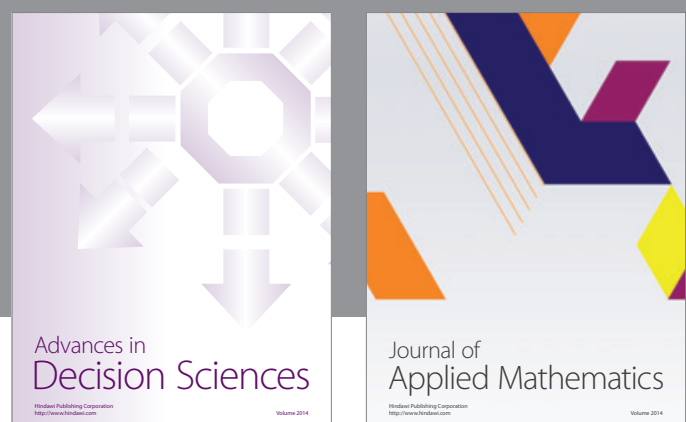

Journal of

Applied Mathematics
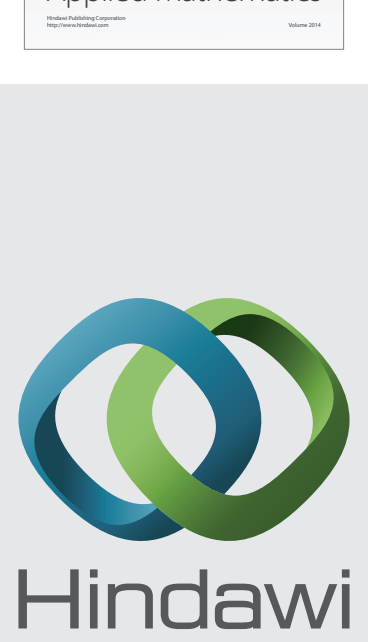

Submit your manuscripts at http://www.hindawi.com
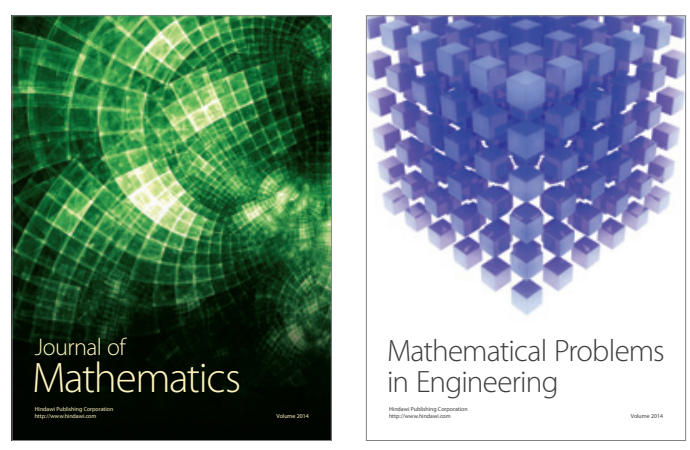

Mathematical Problems in Engineering
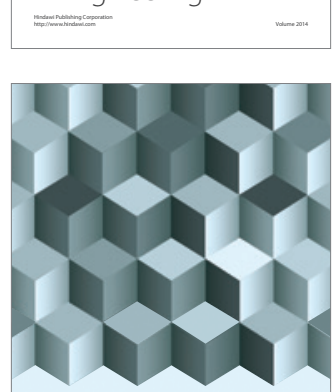

Journal of

Function Spaces
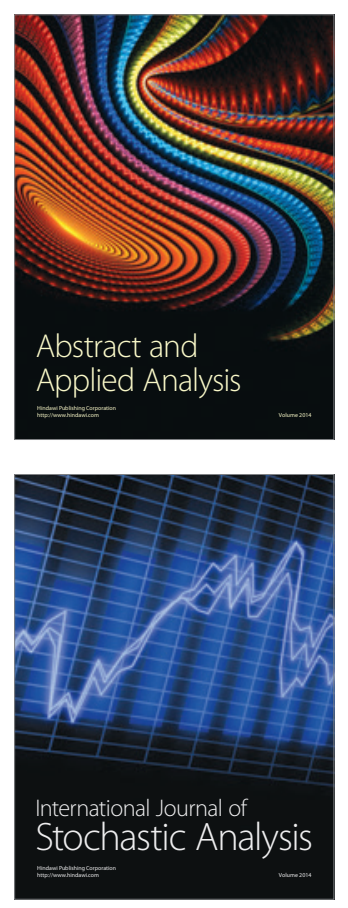

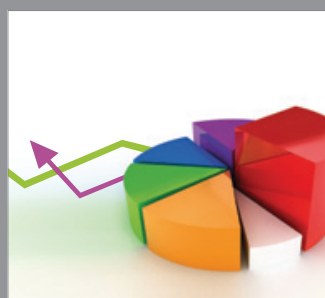

ournal of

Probability and Statistics

Promensencen
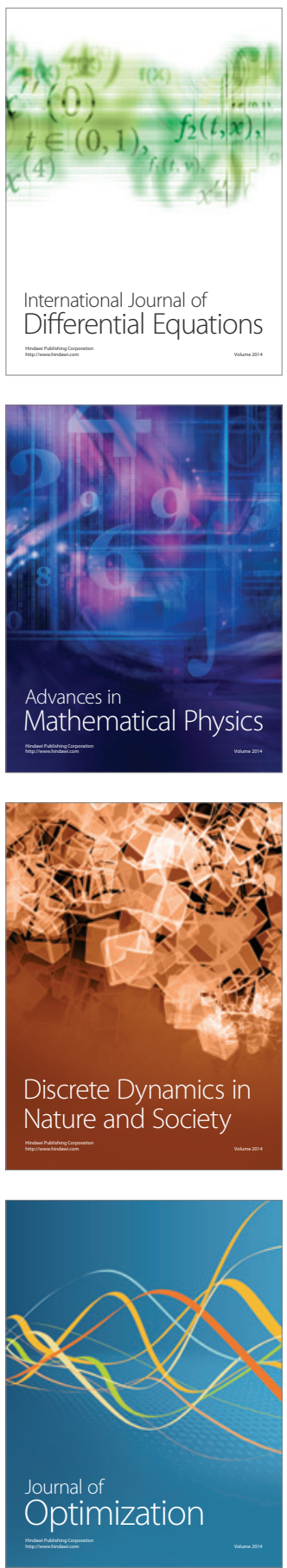